INPLASY

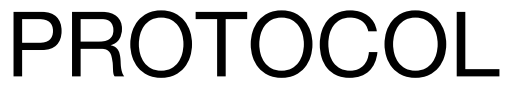

To cite: Wu et al. Effects of mindfulness-based cognitive therapy on current depressive symptoms in older adults: a meta-analysis. Inplasy protocol 202210121. doi:

10.37766/inplasy2022.1.0121

Received: 26 January 2022

Published: 26 January 2022

Corresponding author:

Aixiang Xiao

543061910@qq.com

Author Affiliation:

Affiliated Brain Hospital of

Guangzhou Medical University.

Support: None.

Review Stage at time of this submission: Data analysis.

Conflicts of interest:

None declared.

\section{Effects of mindfulness-based cognitive therapy on current depressive symptoms in older adults: a meta-analysis}

Wu, C1; Ye, J2; Xiao, A3; Guo, J4; Yu, L5; Zhong, X6; Wu, S7; Wang, $\mathrm{H}^{8}$; Wang, T9; Yuan, L10.

Review question / Objective: The aim of this literature review study was to examine the effectiveness of MBCT in older adults with late-life depression.

Rationale: Mindfulness-based cognitive therapy (MBCT), a combination of mindfulness-based stress reduction and cognitive behavioral therapy, has been designed to prevent relapse for elderly patients with depression in remission. The benefits of MBCT for younger adults in recovering from depression are indicated by its use in clinical practice and numerous empirical studies. However, no recent systematic review has explored the effectiveness of MBCT for use with older adults with depression.

INPLASY registration number: This protocol was registered with the International Platform of Registered Systematic Review and Meta-Analysis Protocols (INPLASY) on 26 January 2022 and was last updated on 26 January 2022 (registration number INPLASY202210121).

\section{INTRODUCTION}

Review question / Objective: The aim of this literature review study was to examine the effectiveness of MBCT in older adults with late-life depression.

Rationale: Mindfulness-based cognitive therapy (MBCT), a combination of mindfulness-based stress reduction and cognitive behavioral therapy, has been designed to prevent relapse for elderly patients with depression in remission. The benefits of MBCT for younger adults in recovering from depression are indicated by its use in clinical practice and numerous empirical studies. However, no recent systematic review has explored the effectiveness of MBCT for use with older adults with depression.

Condition being studied: Effects of mindfulness-based cognitive therapy on current depressive symptoms in older 
adults. There are related equipment and personnel support.

\section{METHODS}

Participant or population: Participants aged 60 years and older who is suffering from depression.

Intervention: Mindfulness-based cognitive therapy ( MBCT).

Comparator: Active or passive control for comparison will both be considered.

Study designs to be included: Randomized controlled study and pre-post study.

Eligibility criteria: Being diagnosed with light to sever depression.

Information sources: Electronic databases, trial registers and manual search of identified systematic review.

Main outcome(s): The effect measures included symptom-specific depression scales such as the Patient Health Questionnaire-9 (PHQ-9) and the Beck Depression Inventory (BDI-II). We also included those using measures of general (rather than specific) depressive symptoms, such as the Depression Anxiety Stress Scales.

Quality assessment / Risk of bias analysis: Due to the variety of study designs, the methodological quality and risk of bias across the included studies were assessed by checking certain items: (a) method of treatment adherence reporting and (b) outcome measurements at follow-up. For controlled studies, we also extracted data on (a) type of data analyses, (b) research design method, (c) whether assessors were blinded regarding the group allocations, and (d) whether the amount of time in treatment was equal across groups.

Strategy of data synthesis: Heterogeneity analysis will be evaluated by $Q$ and $\mathbf{I 2}$ indicators, if $Q$ is significant and $I 2 \geq 75 \%$, it indicates that there is non-negligible heterogeneity between studies, we will choose a random effect model to syntesize, or with a fixed effect model. All data will be analyzed using Comprehensive MetaAnalysis software (CMA). Using means, standard deviations, and sample size to calculate Hedge's $g$ as the effect size.

Subgroup analysis: Not Available.

Sensitivity analysis: Not Available.

Language: English only.

Country(ies) involved: China.

Keywords: depression; mindfulness-based cognitive therapy (MBCT); older adults.

Contributions of each author:

Author 1 - Chenxin Wu - The author drafted the manuscript and participated in data collection and process.

Email: 975911601@qq.com

Author 2 - Junrong Ye - The author conceived the original idea of this study and drafted the manuscript.

Email: yejunrong1580@qq.com

Author 3 - Aixiang Xiao - The author conceived the original idea of this study and amended the manuscript.

Email: 543061910@qq.com

Author 4 - Jianxiong Guo - The author amended the manuscript and participated in data statistical analysis.

Email: jxguonet01@126.com

Author 5 - Lin Yu - The author amended the manuscript and participated in data statistical analysis.

Email: yulinfimmu@126.com

Author 6 - Xiaomei Zhong - The author conceived the original idea of this study.

Email:

Author 7 - Shengwei Wu - The author participated in data collection and process and amended the manuscript.

Author 8 - Haoyun Wang - The author participated in data collection and process.

Author 9 - Ting Wang - The author participated in data collection and process. Author 10 - Lexin Yuan - The author participated in data collection and process. 